\title{
Aquaporin-dependent excessive intrauterine fluid accumulation is a major contributor in hyper-estrogen induced aberrant embryo implantation
}

Cell Research (2015) 25:139-142. doi:10.1038/cr.2014.139; published online 24 October 2014

\section{Dear Editor,}

In mammalian species, the window of implantation is defined as a limited period when implantation-competent blastocyst can interact with the receptive endometrium [1]. Besides the acquisition of blastocyst competency and uterine receptivity, initiation of embryo implantation also involves a timely reabsorption of intrauterine fluids, which facilitates the apposition of embryo with the uterine lining [2]. These processes are coordinated by preimplantation ovarian estrogen (E2) and progesterone (P4): a small E2 surge imposed on a P4-primed environment initiates implantation [1], where the level of E2 and ratio of $\mathrm{P} 4 / \mathrm{E} 2$ must be kept within an optimal range $[3,4]$. To date, numerous genes regulating uterine receptivity and blastocyst implantation have been identified using transgenic mouse models [5]. However, genes controlling uterine fluid homeostasis are much less explored, and their pathophysiological significance in embryo implantation remains poorly understood. In the present study, combining genetic and pharmacological approaches, we demonstrate that an excess of intrauterine fluids caused by increased expression of two water channel genes, aquaporin-5 (Aqp5) and -8 (Aqp8), is a major contributor to abnormal implantation in pregnant mice treated with supraphysiological doses of E2. We also showed that $\mathrm{P} 4$ administration neutralized E2-induced Aqp 5/8 overexpression, preventing excessive intrauterine fluid accumulation and improving implantation.

During the in vitro fertilization/embryo transfer (IVF/ ET) procedure, the standard ovarian hyperstimulation could cause supraphysiological levels of steroid hormones such as E2, leading to altered E2/P4 ratio and subsequently impaired implantation [4]. As uterine fluid homeostasis is dynamically regulated by sterioid hormones (E2 and P4) [2] and an excess of intrauterine fluids at preimplantation causes abnormal implantation $[6,7]$, we hypothesized that abnormal uterine fluid secretion caused by elevated E2 levels may contribute to the defective implantation and aimed to identify the underlying mechanisms. To investigate the influence of supraphysiological E2 levels on embryo implantation, we treated pregnant mice with E2 (with different dosages, Figure 1A) on day 4 of pregnancy (08:30), and found that a single injection of $>50 \mathrm{ng}$ of E2 efficiently disrupted embryo implantation as examined on day 5 and day 6 , showing both delayed implantation and aberrant embryo spacing (Figure 1A-1C). The aberrant implantation indeed led to increased pregnancy loss at midgestation and decreased litter size on day 12 of pregnancy (Figure 1D and Supplementary information, Figure S1A-S1D), consistent with previous reports that aberrant timing and site of implantation caused adverse ripple effects for the ongoing pregnancy [8-10]. We next used this hyper-estrogen-induced pathophysiological mouse model to explore the role of excessive uterine fluid accumulation in the defective implantation. To estimate the volume of intrauterine fluids in mice, the cervix end of a uterine horn was first ligated (on day 3 08:30) to prevent fluid leakage. Twenty-four hours later the mice were treated with either E2 (100 ng) or vehicle (oil) for $8 \mathrm{~h}$, and then the uteri from both groups were dissected for luminal fluid volume measurement (Figure 1E and Supplementary information, Figure S1E). The volume of intrauterine fluids was estimated by the size of the fluid-drop stain on a filter paper (Figure 1F and Supplementary information, Data S1). The uteri from E2-treated mice showed a dramatic increase in the intrauterine fluid volume, while the percentage of water weight within the uterine tissue of E2-treated mice (after releasing the luminal fluid) was similar to that of the control group (Figure 1G), suggesting that fluid retention occurred in the uterine lumen but not within the stroma, which is most likely due to increased fluid net flow into the lumen upon changed interstitial versus intraluminal osmolarity. We further examined the implantation status after performing the same ligation procedure (on day 3) followed by E2 or vehicle treatment (on day 4) (Supplementary information, Figure 
$\mathrm{S} 1 \mathrm{~F}$ ), and found that although the ligation procedure did not hamper embryo implantation in vehicle-treated control group, it enhanced the adverse effect of E2 treatment and caused almost complete implantation failure in the ligated uterine horn (Figure 1H and 1I). These results clearly demonstrated that hyper-estrogen-induced excessive intrauterine fluid retention is an important contributor to defective implantation.
To explore the underlying mechanism for the substantial increase in luminal fluid volume, we performed microarray analyses of E2- and vehicle-treated uteri and searched for potential genes responsible for the drastic intrauterine fluid increase. Among the possible candidates, two water channel genes, Aqp5 and Aqp 8, showed a simultaneous increase in expression levels. As aquaporin-mediated fluid transport is involved in a wide
A

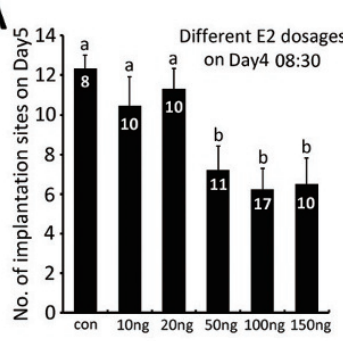

B
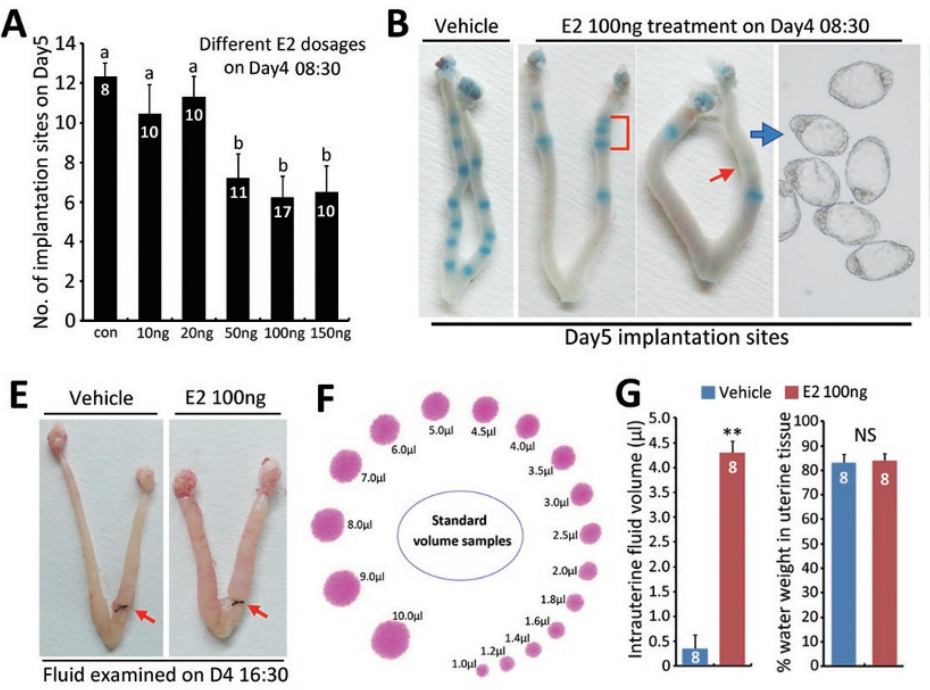

J

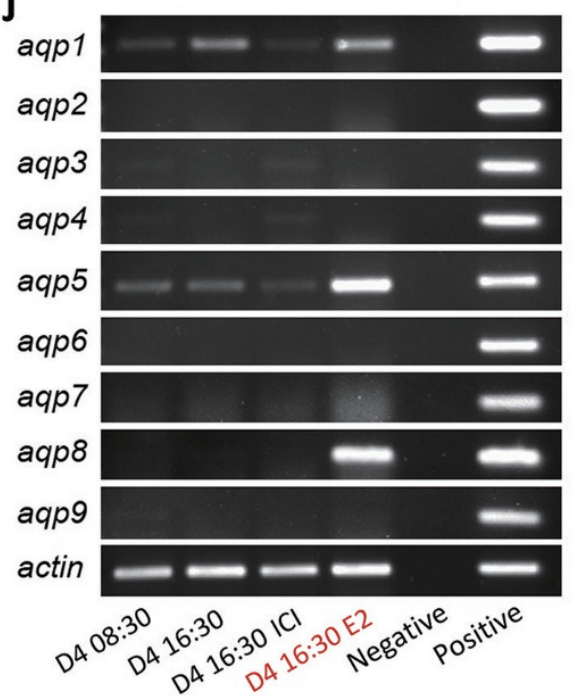

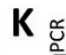
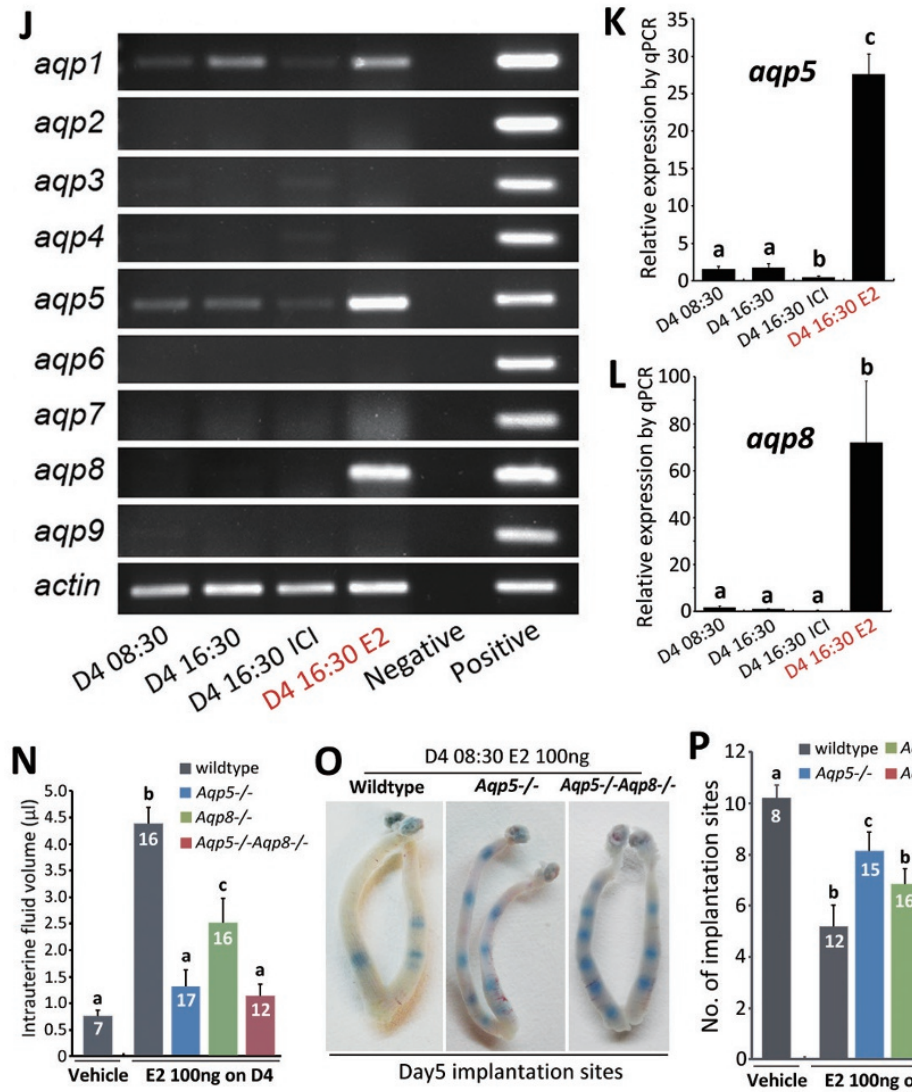

.

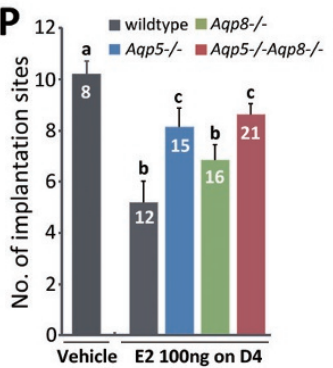

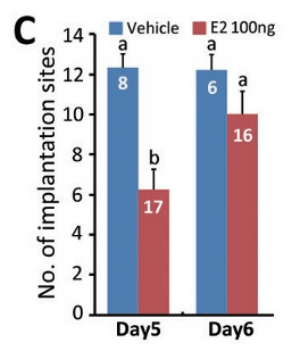

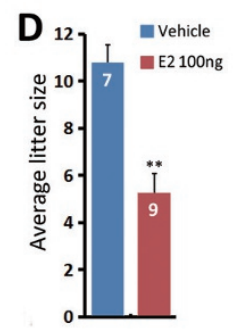

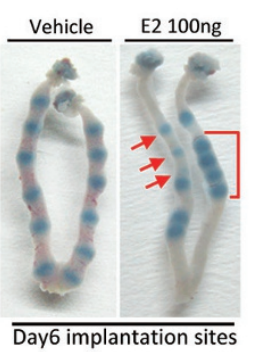
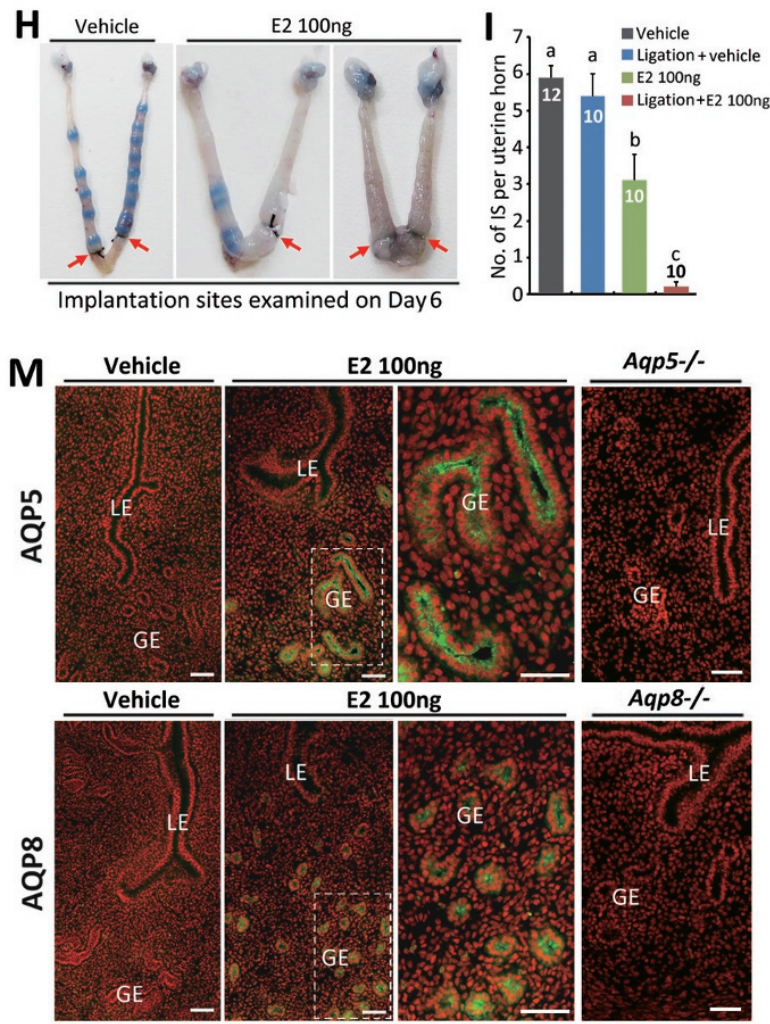

Q

E2 100ng E2 100ng + P4 2mg

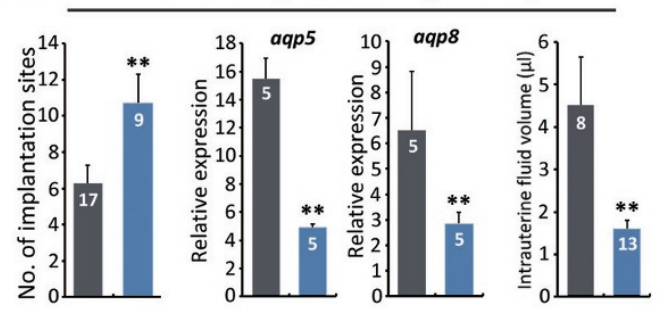


Figure 1 Aqp5/8-dependent excessive intrauterine fluid accumulation contributes to hyper-E2-induced implantation failure. (A) Dose-dependent E2 effects on implantation. (B) Representative pictures of normal and abnormal implantation determined by the blue dye method (days 5 and 6). Each blue band indicates an implantation site. E2-treated group showed delayed implantation (red arrows) and crowded implantation sites (red brackets). (C) Number of implantation sites examined on days 5 and 6 revealed delayed implantation after E2 treatment. The bars with different letters are significantly different $(P<0.01)(D)$ Average litter size ( ${ }^{* *} P<0.01$; student's $t$ test). (E) Representative pictures of ligated uteri before intrauterine fluid release. Red arrows indicate ligation sites. (F) Standardized fluid-drop stains on a filter paper with defined volumes. (G) Intrauterine fluid volume and water weight percentage in uterine tissues. Water weight percentage $=$ (wet uterus weight - dry uterus weight)/wet uterus weight $\times 100 \%$ (see detailed calculation in Supplementary information, Data S1). ${ }^{* *} P<0.01, \mathrm{NS}, P>0.05$; student's $t$ test. $(\mathbf{H}, \mathbf{I})$ Representative pictures $\mathbf{( H )}$ and statistics (I) of implantation sites in ligated/unligated uteri with/without E2 treatment. Red arrows indicate ligation sites. The bars with different letters are significantly different $(P<0.01)$. (J) Expression profiles of aquaporin family members (Aqp1-9) in normal uteri from day 4 morning (D4 08:30), afternoon (D4 16:30) and from mice pretreated with ER antagonist ICI 182780 (D4 16:30 ICI) or E2 $100 \mathrm{ng}$ (D4 16:30 E2). (K, L) Analysis of Aqp5 (K) and Aqp8 (L) expression by real-time PCR. Data shown represent results from 5 independent experiments. The bars with different letters are significantly different $(P<0.01)$. (M) Immunofluorescence examination of Aqp5 and Aqp8 in day 4 16:30 uteri from wild-type mice with/without E2 treatment and from Aqp $5^{-1}$ and Aqp $8^{-1-}$ mice with E2 treatment. Green staining indicates Aqp5 and Aqp8 expression and red staining represents propidium iodide-labeled nuclei. LE, luminal epithelium; GE, glandular epithelium. Scale bars, $100 \mu \mathrm{m}$. (N) Genetic ablation of Aqp5 and/or Aqp8 limited intrauterine fluid accumulation after E2 treatment. The bars with different letters are significantly different $(P<0.01)$. (O, P) Representative pictures $(\mathbf{O})$ and statistics (P) showing that genetic ablation of Aqp5 and/or Aqp8 improves implantation after E2 treatment. The bars with different letters are significantly different $(P<0.01)$. (Q) P4 co-injection neutralized the adverse effects of E2 on Aqp5 and Aqp8 expression, intrauterine fluid volume and number of implantation sites. All error bars represent SEM. Numbers within/above bars indicate the number of mice/uteri examined.

range of physiological and pathological conditions [11], and previous studies have shown that the expression of aquaporins is dynamically regulated in the uterus during pregnancy [12-14], we next examined the uterine expression levels of aquaporin family members in day-4 uteri (untreated, treated with E2 or ER antagonist ICI 182780) by RT-PCR analysis and confirmed the drastic increase in the expression levels of Aqp5 and Aqp 8 after E2 treatment (Figure 1J). A weak expression of Aqp5 was observed in the absence of E2 treatment, which was inhibited by ICI treatment (Figure 1J). Similar results were obtained using real-time PCR analysis (Figure $1 \mathrm{~K}$ and 1L). The expression levels of Aqp $5 / 8$ showed a gradual increase, which coincides with the increase in the volume of intrauterine fluids after E2 treatment (Supplementary information, Figure S1G-S1I). We next performed immunofluorescence analysis for Aqp5 and Aqp8 proteins (antibody specificity was confirmed in positive tissues, Supplementary information, Figure S1J and S1K) in day-4 uteri and observed an enhanced staining of Aqp5 and Aqp8 after E2 treatment (Figure 1M). Notably, both Aqp5 and Aqp8 proteins are specifically localized on the apical surface, the secretion side of the uterine glandular epithelium (Figure 1M), suggesting an active role in E2-induced luminal fluid secretion.

To examine whether Aqp5 and Aqp8 are responsible for the E2-induced excessive luminal fluid accumulation, we bred $A q p 5^{-/}, A q p 8^{--\alpha}$ and $A q p 5^{-/} A q p 8^{-/}$mice, and mated the female knockout (or double-knockout) mice of each strain with wild-type males. Under normal condition, each knockout strain showed normal implantation. The pregnant females were then subjected to E2 treatment $(08: 30$, day 4$)$ for $8 \mathrm{~h}$, followed by uterine immunofluorescence detection of Aqp5/8 (16:30, day 4), intrauterine fluid volume measurement (16:30, day 4) and implantation site examination (08:30, day 5). Upon E2 treatment, both $A q p 5^{--}$and Aqp $8^{--\alpha}$ mice showed significantly decreased luminal fluid volume compared with wild-type mice, and an even greater decrease was observed in $A q p 5^{--\alpha} A q p 8^{--}$uteri (Figure $1 \mathrm{~N}$ ). The number of implantation sites in $A q p 5^{--}$and $A q p 5^{--} A q p 8^{--\alpha}$ mice was also significantly increased compared with the wild type (Figure $1 \mathrm{O}$ and 1P). However, even in the Aqp $5^{-/}$Aqp $8^{-/-}$ uteri, the excess of luminal fluid was not completely prevented and the number of implantation sites was not fully restored, which is not surprising as E2 treatment also affects the expression of other ion channels and genes involved in regulating uterine receptivity and embryo implantation [15]. Nonetheless, the substantial correction of excessive luminal fluid retention and the increase in implantation rates in mice lacking Aqp5/8 suggest that these two aquaporins are responsible for E2-induced abnormal luminal fluid accumulation.

Previous reports have shown that a decreased ratio of $\mathrm{P} 4 / \mathrm{E} 2$ is a predictor of human implantation failure, and P4 supplementation could neutralize the adverse effects of elevated E2 level in mouse model [4]. Similarly, improved implantation rate caused by $\mathrm{P} 4$ treatment was confirmed in our experimental system (Figure 1Q), and we further demonstrated that co-injection of $\mathrm{P} 4$ along 
with E2 significantly decreased E2-induced upregulation of Aqp5 and Aqp 8 expression, and reduced the excessive luminal fluid volume (Figure 1Q), which in our belief, is a major contributing factor to the improved implantation.

In summary, the present study demonstrates a novel mechanism that Aqp5/8-dependent excessive intrauterine fluid accumulation is a major contributor to the supraphysiological level of E2-induced aberrant embryo implantation, which represents a critical cause of implantation failure aside from the well-recognized factors of endometrial receptivity. The driving force behind the excessive water movement is an important issue that warrants further investigation. Our data also showed that manipulation of the P4/E2 ratio could improve implantation rate through controlling uterine Aqp5/8-dependent intrauterine fluid accumulation, thus providing a molecular basis for improving IVF-ET outcomes.

\section{Acknowledgments}

This work was supported by the National Basic Research Program of China (2011CB944400, 2011CB710905, 2012CB944702 and 2015CB943000), the Strategic Priority Research Program of the Chinese Academy of Sciences (XDA04020202-20 and XDA04020419) and the National Natural Science Foundation of China (31200879 and 31300957).

Ying Zhang ${ }^{1, *}$, Qi Chen ${ }^{1, *}$, He Zhang ${ }^{1,3,4, *}$, Qiang Wang ${ }^{1, *}$, Rong $\mathrm{Li}^{2}$, Yaping $\mathrm{Jin}^{4}$, Haibin Wang ${ }^{1}$, Tonghui $\mathrm{Ma}^{3}$, Jie Qiao ${ }^{2}$, Enkui Duan ${ }^{1}$
${ }^{I}$ State Key Laboratory of Reproductive Biology, Institute of Zoology, Chinese Academy of Sciences, Beijing 100101, China $;{ }^{2}$ Center of Reproductive Medicine, Department of Obstetrics and Gynecology, Peking University Third Hospital, Beijing 100191, China; ${ }^{3}$ College of Basic Medical Sciences, Dalian Medical University, Dalian, Liaoning 116044, China; ${ }^{4}$ College of Veterinary Medicine, Northwest A\&F University, Yangling, Shaanxi 712100, China

*These four authors contributed equally to this work.

Correspondence: Enkui Duan ${ }^{\mathrm{a}}$, Jie Qiao ${ }^{\mathrm{b}}$, Tonghui $\mathrm{Ma}^{\mathrm{c}}$

${ }^{a}$ Tel: 86-10-64807182

E-mail: duane@ioz.ac.cn

bE-mail: jie.qiao@263.net

cE-mail: tonghuima@dlmedu.edu.cn

\section{References}

1 Cha J, Sun X, Dey SK. Nat Med 2012; 18:1754-1767.

2 Chen Q, Zhang Y, Elad D, et al. Mol Aspects Med 2013; 34:10241042.

3 Ma WG, Song H, Das SK, et al. Proc Natl Acad Sci USA 2003; 100:2963-2968.

4 Gidley-Baird AA, O’Neill C, Sinosich MJ, et al. Fertil Steril 1986; 45:69-74.

5 Zhang S, Lin H, Kong S, et al. Mol Aspects Med 2013; 34:939-980.

6 Akman MA, Erden HF, Bahceci M. Hum Reprod 2005; 20:906-909.

7 Lu S, Peng H, Zhang H, et al. PLoS One 2013; 8:e78446.

8 Chen Q, Zhang Y, Peng H, et al. J Biol Chem 2011; 286:4349-4356.

9 Ye X, Hama K, Contos JJ, et al. Nature 2005; 435:104-108.

10 Song H, Lim H, Paria BC, et al. Development 2002; 129:2879-2889.

11 Verkman AS. Annu Rev Med 2012; 63:303-316.

12 Lindsay LA, Murphy CR. Reprod Fertil Dev 2014; 26:982-990.

13 Lindsay LA, Murphy CR. J Mol Histol 2007; 38:87-95.

14 Richard C, Gao J, Brown N, et al. Endocrinology 2003; 144:15331541.

15 Ruan YC, Chen H, Chan HC. Hum Reprod Update 2014; 20:517-529.

(Supplementary information is linked to the online version of the paper on the Cell Research website.) 